\title{
Variabilidade em população base de pimenteiras ornamentais (Capsicum annuum $\mathrm{L} ._{\text {. }}{ }^{1}$
}

\author{
João José da Silva Neto ${ }^{2}$ Elizanilda Ramalho do Rêgo $o^{3}$, Mayana Ferreira Nascimento ${ }^{4}$, \\ Vital Antônio Lucena Silva Filho ${ }^{5}$, Jorge Xavier de Almeida Neto ${ }^{6}$, Mailson Monteiro do Rêgo
}

\section{RESUMO}

O objetivo deste trabalho foi caracterizar progênies de uma população base de pimenteiras (Capsicum annuum L.). Foram utilizadas 54 plantas de uma geração $\mathrm{F}_{2}$ de pimenteiras ornamentais, para se avaliarem os seguintes caracteres morfoagronômicos: altura da planta (AP), diâmetro da copa (DDC), altura da primeira bifurcação (APB), diâmetro do caule (DCL), comprimento da folha (CFL), largura da folha (LFL), comprimento da corola (CDC), comprimento de antera (CANT), comprimento do estilete (CES) e largura da pétala (LAP). O delineamento experimental foi inteiramente casualizado, com três repetições. Os dados foram submetidos à Análise de Variância, com posterior agrupamento das médias para o teste de Scott-Knott ( $\mathrm{p}<0,01$ ). Com exceção do CANT, verificaram-se diferenças significativas para os demais descritores, pelo Teste F. O DCL foi o descritor com maior herdabilidade $(99,49 \%)$, seguido de DDC (96,14\%) e APB (94,05\%). O caractere DCL apresentou maior variabilidade entre as plantas, formando 13 classes, seguido de DDC, com oito e APB, com seis classes. Quando se utilizaram as técnicas multivariadas, foi possível agrupar as 54 plantas em oito grupos, sendo o grupo 1 o que reuniu maior número de plantas (35). A característica que mais contribuiu para a divergência genética foi o diâmetro do caule $(68,97 \%)$, seguida pelo diâmetro da copa $(9,22 \%)$, altura da primeira bifurcação $(6,76 \%)$ e altura da planta $(4,58 \%)$. Já as características de flor foram as que menos contribuíram para a variabilidade $(10,47 \%)$. Houve variabilidade entre as plantas estudadas, sendo possível praticar seleção dentro dessa família, dando continuidade ao Programa de Melhoramento de Pimenteiras.

Palavras-chave: melhoramento, plantas ornamentais, banco de germoplasma, caracteres morfoagronômicos.

\section{ABSTRACT}

\section{Variability in a base population of pepper (Capsicum annuum L.)}

The objective of this study was to characterize genotypes of a base population of pepper (Capsicum annuит L.). We used 54 plants of a F2 generation of ornamental peppers based on the following morphological characteristics: plant height (PH), canopy width (DDC), first bifurcation height (APB), stem width (DCL), stem length (CFL), leaf length, leaf width (LFL), corolla length (CDC), anther length (CANT), style length (CES) and petal width (LAP). The experimental design was in a completely randomized with three replications. Data were analyzed by ANOVA with subsequent grouping by Scott-Knott criteria ( $\mathrm{p} \mathrm{d}$ " 0.01). Except for CANT, there were significant differences for all the other descriptors by $\mathrm{F}$ test (p d" 0.01). LTB is the descriptor with the highest heritability (99.49\%), followed by DDC (96.14\%) and ABS (94.05\%). The trait DCL showed the greatest variability among the plants, forming 13 groups, followed

Recebido para publicação em 04/03/2013 e aprovado em 14/06/2013.

${ }^{1}$ Este trabalho é parte da dissertação de mestrado do primeiro autor. Financiamento: CNPq (Processo número: 473897/2010-5).

²Engenheiro-Agrônomo. Departamento de Biologia, Universidade Federal da Paraíba, Campus II, Cidade Universitária s/n, 58397-000, Areia, Paraíba, Brasil. netonix @ hotmail.com ${ }^{3}$ Bióloga, Doutora. Departamento de Ciências Fundamentais e Sociais, Universidade Federal da Paraíba, Campus II, Cidade Universitária s/n, 58397-000, Areia, Paraíba, Brasil. elizanilda@cca.ufpb.br (autora parar correspondência).

${ }^{4}$ Bióloga. Departamento de Fitotecnia, Universidade Federal de Viçosa, Campus Viçosa, Avenida Peter Henry Rolfs, s/n, 36570-000, Viçosa, Minas Gerais, Brasil. mayana_nana@hotmail.com

${ }^{5}$ Graduando em Agronomia. Universidade Federal da Paraíba, Campus II, Cidade Universitária s/n, 58397-000, Areia, Paraíba, Brasil. vitallucenna@hotmail.com

${ }^{6}$ Biólogo, Mestre. Departamento de Biologia, Universidade Federal da Paraíba, Campus II, Cidade Universitária s/n, 58397-000, Areia, Paraíba, Brasil. netobiologia2@hotmail.com ${ }^{7}$ Biólogo, Doutor. Departamento de Biologia, Universidade Federal da Paraíba, Campus II, Cidade Universitária s/n, 58397-000, Areia, Paraíba, Brasil. mailson@cca.ufpb.br 
by DDC with 8 and APB with 6 groups. Using multivariate analysis it was possible to separate the 54 plants in eight groups. The group 1 was the largest one with 35 genotypes. The stem width (68.97\%), canopy width (9.22\%), first bifurcation height $(6.76 \%)$ and plant height $(4.58 \%)$ had the highest contribution to the variability. The flower traits had the lowest contribution to the variability $(10.47 \%)$. The results showed that there was variability among genotypes, and then it is possible to perform selection within this family to continue the breeding program of ornamental pepper.

Key words: breeding, ornamental plants, genebank, chili pepper.

\section{INTRODUÇÃO}

As pimentas foram, possivelmente, os primeiros aditivos alimentares utilizados pelas civilizações antigas do México e da América do Sul. Suas características atribuem aroma, cor e sabor aos alimentos, tornando-os mais atraentes (Stommel \& Bosland, 2007). Além de estimulantes do apetite e auxiliares da digestão, os frutos de Capsicum spp. são fontes importantes de três antioxidantes naturais: vitamina $\mathrm{C}$, carotenoides e vitamina $\mathrm{E}$ (Rêgo et al., 2010; Rêgo et al., 2011a; Rêgo et al. 2011b; Rêgo et al, 2012a). Nota-se também que, pela grande diversidade observada para diversas características, como porte, folhagem e coloração dos frutos, tem ocorrido a inserção das pimentas no mercado de plantas ornamentais (Rêgo et al., 2009; Finger et al., 2012).

O agronegócio de pimentas (Capsicum spp.) está entre os melhores exemplos de integração entre todos os que atuam na cadeia produtiva de hortaliças (Ohara \& Pinto, 2012). Segundo Finger et al. (2012), a agricultura familiar tem sido a principal responsável, no Brasil, pela expansão da área cultivada de pimentas. Rêgo et al. (2012b) demonstraram que a produção de novas variedades de pimenteiras ornamentais permitiu o incremento na renda de agricultoras familiares do Estado da Paraíba, propiciando a geração de novos empregos e a fixação dessas agricultoras rurais e de suas famílias, no campo.

A caracterização de germoplasma visa a descrever os diversos acessos de uma coleção, por meio de características de interesse, como a produtividade, a massa de frutos, a espessura de polpa, o número de sementes por fruto, a resistência a pragas e doenças, dentre outras. A partir deste passo, e com o uso de metodologias genéticas e estatísticas, é possível analisar a variabilidade dos diferentes acessos e avaliar seu potencial de uso em programas de melhoramento (Marim et al., 2009). Rêgo et al. (2011a) relataram diversos estudos de variabilidade em pimenteira, baseados tanto em características de porte (altura da planta, diâmetro da copa e altura da primeira bifurcação), como para característica de frutos (comprimento, diâmetro, espessura do pericarpo, matéria fresca, teor de matéria seca, sólidos solúveis totais, teor de vitamina $\mathrm{C}$, dentre outros) desenvolvidos em anos recentes. A Universidade Federal da Paraíba possui um programa de melhoramento de pimenteiras ornamentais, que vem sendo desenvolvido nos últimos doze anos, por meio de hibridação e seleção em gerações segregantes (Rêgo et al., 2012c). O conhecimento da variabilidade dessas gerações é de suma importância para a continuidade do programa (Nascimento et al., 2012; Barroso et al.; 2012).

Desta forma, o melhoramento genético atua como um elo importante na cadeia do agronegócio das plantas ornamentais, em busca de selecionar cultivares resistentes a pragas, doenças, estresses bióticos e abióticos (Stommel \& Bosland, 2007) e com características, como tamanho, precocidade, qualidade dos frutos, capacidade e longevidade do valor ornamental (Rêgo et al., 2011a; Finger et al., 2012). As informações geradas por este trabalho serão úteis, tanto para o programa de melhoramento da UFPB, como para outros pesquisadores que trabalham com o gênero Capsicum. De fato, no programa de melhoramento de pimenteiras ornamentais da UFPB, já foi possível selecionar linhagens com maior tempo de vida pós-produção (Rêgo et al., 2010) e linhas com maior resistência ao etileno (Santos et al., 2013), bem como desenvolver 30 híbridos intraespecíficos $(C$. annиum) (Rêgo et al, 2012d), que geraram diversas famílias $F_{2}$ que se encontram em fase de avaliação. Neste contexto, este estudo teve como objetivo avaliar a diversidade entre progênies desses híbridos, uma família $\mathrm{F}_{2}$ (população base) de pimenteiras ornamentais (Capsicum annuum).

\section{MATERIAL E MÉTODOS}

O experimento foi realizado em casa de vegetação, no Laboratório de Biotecnologia Vegetal, do Centro de Ciências Agrárias da Universidade Federal da Paraíba (CCAUFPB), no município de Areia - PB, em altitude de $618 \mathrm{~m}$, latitude de $06^{\circ} 57^{\prime} 48^{\prime \prime}$ 'S e longitude de $35^{\circ} 41^{\prime} 30^{\prime \prime} \mathrm{O}$. 
Os tratamentos foram constituídos de 54 progênies, de uma geração $\mathrm{F}_{2}$ de pimenteiras ornamentais (Capsicum аппиит), pertencentes ao Banco de Germoplasma do gênero Capsicum, da UFPB, provenientes da autofecundação controlada da $\mathrm{F}_{1}$ (Rêgo et al., 2012b) e obtida do cruzamento entre os genitores UFPB390 x UFPB137, sendo as plantas cultivadas em vasos de $700 \mathrm{~mL}$ preenchidos com substrato comercial.

Para a caracterização morfoagronômica, foram considerados dez caracteres quantitativos: altura da planta (AP), diâmetro da copa (DDC), altura da primeira bifurcação (APB), diâmetro do caule (DCL), comprimento da folha (CFL), largura da folha (LDF), comprimento da corola (CDC), comprimento da antera (CANT), comprimento do estilete (CES), largura da pétala (LAP), conforme a lista de descritores sugeridos pelo IPGRI (1995). Todas as medidas foram expressas em centímetros.

Para cálculo das variâncias residuais, utilizaram-se três testemunhas adicionais (Cruz, 2008). O experimento foi analisado no delineamento inteiramente casualizado, com três repetições de 18 plantas. Os dados foram submetidos à análise de variância e as médias foram agrupadas pelo teste de Scott-Knott, a 1\% de probabilidade.

Também foram calculadas as estimativas da herdabilidade, no sentido amplo, $\left(h^{2}\right)$, dada pelo seguinte estimador:

$\mathrm{h}^{2}=\frac{\hat{\sigma}_{g}^{2}}{\hat{\sigma}_{p}{ }^{2}} \times 100$,

em que: ${ }_{g} \hat{\sigma}^{2}=$ variância genética;

$\hat{\sigma}_{p}^{2}=$ variância fenotípica.

A variância genética foi calculada pelo estimador:

$\hat{\sigma}_{g}^{2}=\frac{Q M \text { trat }-Q M r e s}{J}$

em que: QMtrat=quadrado médio do tratamento;

QMres= quadrado médio do resíduo;
$\mathrm{J}=$ número de repetições.

Para análise de divergência genética, utilizou-se o método de Tocher, com base na distância generalizada de Mahalanobis. A importância relativa das características foi determinada, utilizando-se o método de Singh (1981). Todas as análises foram realizadas com o programa computacional Genes (Cruz, 2008).

\section{RESULTADOS E DISCUSSÃO}

Verificou-se a ocorrência de diferenças significativas ( $\mathrm{p}$ $<0,01)$ para todos os caracteres analisados, exceto para o comprimento da antera (Tabela 1). Nascimento et al. (2012), trabalhando com outra família $\mathrm{F}_{2}$ de $C$. аnnиum, encontraram significância para esta mesma característica. Este fato evidencia a presença de diversidade genética entre as famílias $\mathrm{F}_{2}$ de $C$. апnиит, ressaltando a necessidade de se realizarem estudos, não só dentro de famílias, como entre as famílias. $\mathrm{O}$ comprimento da antera também apresentou o menor valor de herdabilidade $(22,25 \%)$ e o maior valor de coeficiente de variação ambiental (CV) 40,83\% (Tabela 1), evidenciando ser esta a característica mais influenciada pelos componentes ambientais, o que dificulta a seleção para a mesma. Rêgo et al. (2012d), trabalhando com híbridos da espécie C. anпиит, também não verificaram variação significativa para esta variável, mesmo tendo, estes autores, encontrado menor valor de CV para essa característica (14,89\%).

Os coeficientes de variação oscilaram de 1,63 a 40,83\%. Por outro lado, os valores de herdabilidade foram altos, acima de $73 \%$, para os demais descritores, exceto para o comprimento da antera (Tabela 1). Altos valores de herdabilidade para características quantitativas também foram encontrados por outros autores (Sreelathakumary \& Rajamony, 2004; Rêgo et al., 2011c; Nascimento et al., 2012). Os altos valores de herdabilidade indicam que a variação encontrada para as variáveis em questão são devidas mais à variação genética do que à variação ambiental, tornando possível o ganho genético, para essas características, por seleção.

Tabela 1. Quadrados médios para dez descritores quantitativos de plantas em pimenteiras ornamentais (Capsicum annuum)

\begin{tabular}{|c|c|c|c|c|c|}
\hline \multirow[t]{2}{*}{ F.V. } & \multicolumn{2}{|r|}{ Quadrado } & \multicolumn{2}{|l|}{ Médio } & \multirow[b]{2}{*}{ CFL } \\
\hline & $\mathbf{A P}$ & DDC & APB & DCL & \\
\hline Tratamento & $43.560 * *$ & $25.901 * *$ & $16.693 * *$ & $0,019 * *$ & $1.111 * *$ \\
\hline $\mathrm{h}^{2}(\%)$ & 92,87 & 96,14 & 94,05 & 99,49 & 78,84 \\
\hline \multirow[t]{2}{*}{$\mathrm{CV}(\%)$} & 7,67 & 3,86 & 9,07 & 1,63 & 12,65 \\
\hline & CDC & CANT & CES & LAP & LDF \\
\hline Tratamento & $0,161 * *$ & $0,008^{\mathrm{ns}}$ & $0,018 * *$ & $0,007 * *$ & $0,199 * *$ \\
\hline $\mathrm{h}^{2}(\%)$ & 85,61 & 22,25 & 92,29 & 82,11 & 73,20 \\
\hline $\mathrm{CV}(\%)$ & 11,98 & 40,83 & 8,06 & 10,06 & 13,20 \\
\hline
\end{tabular}

AP - Altura da Planta (cm); DDC - Diâmetro da Copa (cm); APB - Altura da Primeira Bifurcação (cm); DCL - Diâmetro do Caule (cm); CFL - Comprimento da Folha (cm); LDF - Largura da Folha (cm); CDC - Comprimento da Corola $(\mathrm{cm})$; CANT - Comprimento da Antera $(\mathrm{cm})$; CES - Comprimento do Estilete $(\mathrm{cm})$; LAP - Largura da Pétala $(\mathrm{cm})$.

Rev. Ceres, Viçosa, v. 61, n.1, p. 084-089, jan/fev, 2014 
Tabela 2. Médias de características morfoagronômicas de 54 plantas de geração F2 de pimenteira ornamental (Capsicum annuum)

\begin{tabular}{|c|c|c|c|c|c|c|c|c|c|c|}
\hline Planta & $\mathbf{A P}$ & DDC & APB & DCL & CFL & LDF & CDC & CANT & CES & LAP \\
\hline 1 & $22 \mathrm{~d}$ & $24 \mathrm{f}$ & $13,6 \mathrm{c}$ & $0,58 \mathrm{~h}$ & $5,27 \mathrm{a}$ & $2,33 \mathrm{a}$ & $1,34 \mathrm{~b}$ & $0,21 \mathrm{a}$ & $0,60 \mathrm{a}$ & $0,39 \mathrm{~b}$ \\
\hline 2 & $26,5 b$ & $24,5 f$ & $14 c$ & $0,65 \mathrm{e}$ & $4,77 \mathrm{a}$ & $2,26 \mathrm{a}$ & $1,43 b$ & $0,19 \mathrm{a}$ & $0,59 \mathrm{a}$ & $0,46 \mathrm{a}$ \\
\hline 3 & $26,5 b$ & $22 \mathrm{~g}$ & $14 \mathrm{c}$ & $0,65 \mathrm{e}$ & $4,33 b$ & $1,86 \mathrm{a}$ & $1,61 \mathrm{a}$ & $0,2 \mathrm{a}$ & $0,63 \mathrm{a}$ & $0,47 \mathrm{a}$ \\
\hline 4 & $18,5 \mathrm{e}$ & $24 \mathrm{f}$ & $8 \mathrm{f}$ & $0,58 \mathrm{~h}$ & $3,56 \mathrm{c}$ & $1,5 b$ & $1,29 \mathrm{c}$ & $0,21 \mathrm{a}$ & $0,57 \mathrm{a}$ & $0,37 b$ \\
\hline 5 & $25 \mathrm{c}$ & $29,5 b$ & $0,3 \mathrm{~d}$ & $0,55 \mathrm{j}$ & $3,13 \mathrm{c}$ & $1,36 \mathrm{~b}$ & $1,81 \mathrm{a}$ & $0,53 \mathrm{a}$ & $0,60 \mathrm{a}$ & $0,45 \mathrm{a}$ \\
\hline 6 & $26,5 b$ & $23 \mathrm{~g}$ & $12 \mathrm{~d}$ & $0,62 f$ & $4,56 \mathrm{a}$ & $2 \mathrm{a}$ & $1,46 b$ & $0,22 \mathrm{a}$ & $0,58 \mathrm{a}$ & $0,41 b$ \\
\hline 7 & $21,5 \mathrm{~d}$ & $26 \mathrm{e}$ & $10,2 \mathrm{e}$ & $0,65 \mathrm{e}$ & $4,26 b$ & $2,3 a$ & $1,69 \mathrm{a}$ & $0,2 \mathrm{a}$ & $0,51 b$ & $0,40 \mathrm{~b}$ \\
\hline 8 & $22,5 \mathrm{~d}$ & $21,5 \mathrm{~h}$ & $14 \mathrm{c}$ & $0,56 \mathrm{i}$ & $3,93 b$ & $1,6 \mathrm{~b}$ & $1,48 b$ & $0,19 a$ & $0,48 \mathrm{c}$ & $0,35 \mathrm{c}$ \\
\hline 9 & $24,5 \mathrm{c}$ & $23 \mathrm{~g}$ & $0,5 \mathrm{~d}$ & $0,64 \mathrm{e}$ & $4,86 a$ & $1,93 \mathrm{a}$ & $1,42 b$ & $0,17 \mathrm{a}$ & $0,57 \mathrm{a}$ & $0,34 \mathrm{c}$ \\
\hline 10 & $17,5 \mathrm{e}$ & $24 \mathrm{f}$ & $8,5 \mathrm{f}$ & $0,6 \mathrm{~g}$ & $3,83 \mathrm{c}$ & $1,9 \mathrm{a}$ & $1,52 b$ & $0,22 \mathrm{a}$ & $0,56 \mathrm{a}$ & $0,41 b$ \\
\hline 11 & $20 \mathrm{~d}$ & $26 \mathrm{e}$ & $10 \mathrm{e}$ & $0,62 f$ & $3,46 \mathrm{c}$ & $1,6 b$ & $1,64 \mathrm{a}$ & $0,2 \mathrm{a}$ & $0,60 \mathrm{a}$ & $0,43 b$ \\
\hline 12 & $18,5 \mathrm{e}$ & $25 f$ & $10 \mathrm{e}$ & $0,64 \mathrm{e}$ & $3,56 \mathrm{c}$ & $1,63 b$ & $1,48 b$ & $0,21 \mathrm{a}$ & $0,55 \mathrm{a}$ & $0,38 b$ \\
\hline 13 & $22,5 d$ & $25 f$ & $12,7 d$ & $0,61 \mathrm{~g}$ & $4,23 b$ & $1,93 \mathrm{a}$ & $1,58 \mathrm{a}$ & $0,2 \mathrm{a}$ & $0,58 \mathrm{a}$ & $0,41 b$ \\
\hline 14 & $26 b$ & $24,5 f$ & $10,5 \mathrm{e}$ & $0,65 \mathrm{e}$ & $3,73 \mathrm{c}$ & $1,86 \mathrm{a}$ & $1,69 \mathrm{a}$ & $0,2 \mathrm{a}$ & $0,60 \mathrm{a}$ & $0,50 \mathrm{a}$ \\
\hline 15 & $22 \mathrm{~d}$ & $24,5 f$ & $10 \mathrm{e}$ & $0,6 \mathrm{~g}$ & $3,9 b$ & $1,73 b$ & $1,55 \mathrm{a}$ & $0,18 \mathrm{a}$ & $0,54 b$ & $0,35 \mathrm{c}$ \\
\hline 16 & $21,2 d$ & $23,5 \mathrm{~g}$ & $12,3 \mathrm{~d}$ & $0,42 \mathrm{~m}$ & $3,8 \mathrm{c}$ & $1,83 \mathrm{a}$ & $1,41 b$ & $0,18 \mathrm{a}$ & $0,51 b$ & $0,40 \mathrm{~b}$ \\
\hline 17 & $19 \mathrm{e}$ & $25 f$ & $8,5 f$ & $0,52 \mathrm{k}$ & $4,3 b$ & $2 \mathrm{a}$ & $1,47 b$ & $0,17 \mathrm{a}$ & $0,43 \mathrm{c}$ & $0,38 b$ \\
\hline 18 & $23 \mathrm{c}$ & $29 \mathrm{c}$ & $13 c$ & $0,71 \mathrm{c}$ & $4,13 b$ & $2 \mathrm{a}$ & $1,33 b$ & $0,19 \mathrm{a}$ & $0,45 \mathrm{c}$ & $0,33 \mathrm{c}$ \\
\hline 19 & $19 \mathrm{e}$ & $26 \mathrm{e}$ & $8,5 \mathrm{f}$ & $0,5 \mathrm{k}$ & $3,6 \mathrm{c}$ & $1,66 b$ & $1,55 \mathrm{a}$ & $0,17 \mathrm{a}$ & $0,53 b$ & $0,41 b$ \\
\hline 20 & $25 c$ & $24 \mathrm{f}$ & $13 c$ & $0,79 b$ & $4,76 \mathrm{a}$ & $1,96 \mathrm{a}$ & $1,23 \mathrm{c}$ & $0,14 \mathrm{a}$ & $0,43 \mathrm{c}$ & $0,38 b$ \\
\hline 21 & $21 \mathrm{~d}$ & $26,5 d$ & $0,0 \mathrm{e}$ & $0,52 \mathrm{k}$ & $4,76 a$ & $2,16 \mathrm{a}$ & $1,21 \mathrm{c}$ & $0,19 \mathrm{a}$ & $0,43 \mathrm{c}$ & $0,33 \mathrm{c}$ \\
\hline 22 & $26,5 b$ & $28 \mathrm{c}$ & $15,5 b$ & $0,54 j$ & $4,23 b$ & $2 \mathrm{a}$ & $1,36 \mathrm{~b}$ & $0,16 \mathrm{a}$ & $0,39 \mathrm{c}$ & $0,38 b$ \\
\hline 23 & $23 \mathrm{c}$ & $26 \mathrm{e}$ & $10,5 \mathrm{e}$ & $0,57 \mathrm{i}$ & $4,7 \mathrm{a}$ & $1,76 \mathrm{~b}$ & $1,31 \mathrm{c}$ & $0,18 \mathrm{a}$ & $0,41 \mathrm{c}$ & $0,33 \mathrm{c}$ \\
\hline 24 & $19 \mathrm{e}$ & $22,5 \mathrm{~g}$ & $12 \mathrm{~d}$ & $0,6 \mathrm{~g}$ & $3,36 \mathrm{c}$ & $1,53 \mathrm{~b}$ & $1,03 \mathrm{c}$ & $0,13 \mathrm{a}$ & $0,4 c$ & $0,31 \mathrm{c}$ \\
\hline 25 & $24 c$ & $27 d$ & $10,5 \mathrm{e}$ & $0,63 f$ & $4 b$ & $1,73 b$ & $1,3 \mathrm{c}$ & $0,23 \mathrm{a}$ & $0,47 \mathrm{c}$ & $0,35 \mathrm{c}$ \\
\hline 26 & $27 b$ & $32 \mathrm{a}$ & $13,5 \mathrm{c}$ & $0,67 d$ & $3,8 \mathrm{c}$ & $1,46 b$ & $1,31 \mathrm{c}$ & $0,19 \mathrm{a}$ & $0,41 \mathrm{c}$ & $0,35 \mathrm{c}$ \\
\hline 27 & $29 \mathrm{a}$ & $26 \mathrm{e}$ & $18 \mathrm{a}$ & $0,65 \mathrm{e}$ & $3 c$ & $1,4 b$ & $1,36 \mathrm{~b}$ & $0,23 \mathrm{a}$ & $0,53 b$ & $0,38 b$ \\
\hline 28 & $26 \mathrm{~b}$ & $30 \mathrm{~b}$ & $12 \mathrm{~d}$ & $0,64 \mathrm{e}$ & $3,06 \mathrm{c}$ & $1,63 b$ & $1,1 \mathrm{c}$ & $0,25 \mathrm{a}$ & $0,44 c$ & $0,35 \mathrm{c}$ \\
\hline 29 & $24 \mathrm{c}$ & $28,5 \mathrm{c}$ & $9 f$ & $0,59 \mathrm{~h}$ & $2,93 \mathrm{c}$ & $1,66 b$ & $1,67 \mathrm{c}$ & $0,20 \mathrm{a}$ & $0,38 \mathrm{c}$ & $0,33 \mathrm{c}$ \\
\hline 30 & $28,5 \mathrm{a}$ & $26 \mathrm{e}$ & $12,5 \mathrm{~d}$ & $0,89 \mathrm{a}$ & $3,23 \mathrm{c}$ & $1,46 b$ & $1,12 \mathrm{c}$ & $0,21 \mathrm{a}$ & $0,41 \mathrm{c}$ & $0,29 \mathrm{c}$ \\
\hline 31 & $25 \mathrm{c}$ & $28 \mathrm{c}$ & $0,0 \mathrm{e}$ & $0,68 \mathrm{~d}$ & $4,03 b$ & $1,9 \mathrm{a}$ & $1,11 \mathrm{c}$ & $0,22 \mathrm{a}$ & $0,41 \mathrm{c}$ & $0,33 \mathrm{c}$ \\
\hline 32 & $29 a$ & $30 \mathrm{~b}$ & $14,33 \mathrm{c}$ & $0,64 \mathrm{e}$ & $3,5 \mathrm{c}$ & $1,4 \mathrm{~b}$ & $1 \mathrm{c}$ & $0,14 \mathrm{a}$ & $0,42 \mathrm{c}$ & $0,32 \mathrm{c}$ \\
\hline 33 & $14 \mathrm{e}$ & $25,5 \mathrm{e}$ & $6 \mathrm{~g}$ & $0,52 \mathrm{k}$ & $3,33 \mathrm{c}$ & $1,66 b$ & $1,20 \mathrm{c}$ & $0,15 \mathrm{a}$ & $0,42 \mathrm{c}$ & $0,32 \mathrm{c}$ \\
\hline 34 & $28 \mathrm{a}$ & $29 \mathrm{c}$ & $10 \mathrm{e}$ & $0,64 \mathrm{e}$ & $3,86 \mathrm{~b}$ & $1,63 b$ & $1,20 \mathrm{c}$ & $0,13 \mathrm{a}$ & $0,42 \mathrm{c}$ & $0,35 \mathrm{c}$ \\
\hline 35 & $24 c$ & $34 \mathrm{a}$ & $8 \mathrm{f}$ & $0,65 \mathrm{e}$ & $3,43 \mathrm{c}$ & $1,5 b$ & $1,19 \mathrm{c}$ & $0,18 \mathrm{a}$ & $0,45 \mathrm{c}$ & $0,34 \mathrm{c}$ \\
\hline 36 & $19,5 \mathrm{~d}$ & $24 f$ & $10,5 \mathrm{e}$ & $0,51 \mathrm{k}$ & $2,3 \mathrm{c}$ & $1,43 b$ & $1,20 \mathrm{c}$ & $0,15 \mathrm{a}$ & $0,5 b$ & $0,35 \mathrm{c}$ \\
\hline 37 & $18 \mathrm{e}$ & $27 \mathrm{~d}$ & $7 \mathrm{~g}$ & $0,55 \mathrm{j}$ & $3,5 \mathrm{c}$ & $1,56 \mathrm{~b}$ & $1,10 \mathrm{c}$ & $0,2 \mathrm{a}$ & $0,4 \mathrm{c}$ & $0,30 \mathrm{c}$ \\
\hline 38 & $31 \mathrm{a}$ & $31 b$ & $13 c$ & $0,7 \mathrm{c}$ & $5,1 \mathrm{a}$ & $2,03 \mathrm{a}$ & $1,09 \mathrm{c}$ & $0,23 \mathrm{a}$ & $0,39 \mathrm{c}$ & $0,30 \mathrm{c}$ \\
\hline 39 & $26 b$ & $24 \mathrm{f}$ & $13,5 \mathrm{c}$ & $0,57 \mathrm{i}$ & $3,56 \mathrm{c}$ & $1,83 \mathrm{a}$ & $0,92 \mathrm{c}$ & $0,2 \mathrm{a}$ & $0,37 \mathrm{c}$ & $0,28 \mathrm{c}$ \\
\hline 40 & $22 \mathrm{~d}$ & $23 \mathrm{~g}$ & $9 \mathrm{f}$ & $0,59 \mathrm{~h}$ & $3,36 \mathrm{c}$ & $1,5 \mathrm{~b}$ & $1,12 \mathrm{c}$ & $0,2 \mathrm{a}$ & $0,35 \mathrm{c}$ & $0,42 b$ \\
\hline 41 & $21 \mathrm{~d}$ & $27 d$ & $9 f$ & $0,66 \mathrm{e}$ & $4,43 b$ & $1,86 a$ & $0,47 d$ & $0,20 \mathrm{a}$ & $0,38 \mathrm{c}$ & $0,30 \mathrm{c}$ \\
\hline 42 & $24,5 \mathrm{c}$ & $28 \mathrm{c}$ & $9 \mathrm{f}$ & $0,67 d$ & $4,06 \mathrm{~b}$ & $1,66 \mathrm{~b}$ & $1,20 \mathrm{c}$ & $0,24 \mathrm{a}$ & $0,49 \mathrm{~b}$ & $0,32 \mathrm{c}$ \\
\hline 43 & $16 \mathrm{e}$ & $23 \mathrm{~g}$ & $6,5 \mathrm{~g}$ & 0,461 & $3,33 \mathrm{c}$ & $1,36 \mathrm{~b}$ & $1,10 \mathrm{c}$ & $0,19 \mathrm{a}$ & $0,47 \mathrm{c}$ & $0,36 \mathrm{c}$ \\
\hline 44 & $23,5 \mathrm{c}$ & $22 \mathrm{~g}$ & $10 \mathrm{e}$ & $0,57 \mathrm{i}$ & $3,43 \mathrm{c}$ & $1,4 \mathrm{~b}$ & $1,06 \mathrm{c}$ & $0,2 \mathrm{a}$ & $0,44 \mathrm{c}$ & $0,29 \mathrm{c}$ \\
\hline 45 & $26 \mathrm{~b}$ & $30 \mathrm{~b}$ & $12,5 \mathrm{~d}$ & $0,6 \mathrm{~g}$ & $4,2 b$ & $1,66 b$ & $0,97 \mathrm{c}$ & $0,19 a$ & $0,4 c$ & $0,32 \mathrm{c}$ \\
\hline 46 & $27 b$ & $26 \mathrm{e}$ & $9,5 \mathrm{e}$ & $0,68 \mathrm{~d}$ & $4,03 b$ & $2,3 a$ & $1,08 \mathrm{c}$ & $0,2 \mathrm{a}$ & $0,41 \mathrm{c}$ & $0,33 \mathrm{c}$ \\
\hline 47 & $20 \mathrm{~d}$ & $22 \mathrm{~g}$ & $12,5 \mathrm{~d}$ & $0,71 \mathrm{c}$ & $3,6 \mathrm{c}$ & $1,93 \mathrm{a}$ & $1,02 \mathrm{c}$ & $0,2 \mathrm{a}$ & $0,44 \mathrm{c}$ & $0,32 \mathrm{c}$ \\
\hline 48 & $17 \mathrm{e}$ & $20 \mathrm{~h}$ & $8,5 \mathrm{f}$ & 0,461 & $2,9 \mathrm{c}$ & $1,63 b$ & $1,22 \mathrm{c}$ & $0,21 \mathrm{a}$ & $0,39 \mathrm{c}$ & $0,29 \mathrm{c}$ \\
\hline 49 & $24 c$ & $25 f$ & $10 \mathrm{e}$ & $0,72 \mathrm{c}$ & $3,06 \mathrm{c}$ & $1,53 b$ & $1,26 \mathrm{c}$ & $0,19 \mathrm{a}$ & $0,41 \mathrm{c}$ & $0,37 \mathrm{c}$ \\
\hline 50 & $21 \mathrm{~d}$ & $27 d$ & 9f & $0,61 \mathrm{~g}$ & $3,66 \mathrm{c}$ & $1,5 b$ & $1,21 \mathrm{c}$ & $0,19 \mathrm{a}$ & $0,39 \mathrm{c}$ & $0,35 \mathrm{c}$ \\
\hline 51 & $22 \mathrm{~d}$ & $21 \mathrm{~h}$ & $0,5 \mathrm{~d}$ & $0,6 \mathrm{~g}$ & $3,16 \mathrm{c}$ & $1,5 \mathrm{~b}$ & $1,24 \mathrm{c}$ & $0,19 \mathrm{a}$ & $0,38 \mathrm{c}$ & $0,34 \mathrm{c}$ \\
\hline 52 & $28,5 \mathrm{a}$ & $27 d$ & $13 c$ & $0,67 d$ & $4 b$ & $1,7 \mathrm{~b}$ & $1,17 \mathrm{c}$ & $0,16 \mathrm{a}$ & $0,38 \mathrm{c}$ & $0,32 \mathrm{c}$ \\
\hline 53 & $16,33 \mathrm{e}$ & $29 \mathrm{c}$ & $10 \mathrm{e}$ & $0,6 \mathrm{~g}$ & $3,93 b$ & $1,9 \mathrm{a}$ & $1,18 \mathrm{c}$ & $0,19 a$ & $0,39 \mathrm{c}$ & $0,36 \mathrm{c}$ \\
\hline 54 & $21 d$ & $28 \mathrm{c}$ & $9,5 \mathrm{e}$ & $0,7 \mathrm{c}$ & $4,06 \mathrm{~b}$ & $1,93 \mathrm{a}$ & $1,06 \mathrm{c}$ & $0,17 \mathrm{a}$ & $0,43 c$ & $0,33 \mathrm{c}$ \\
\hline
\end{tabular}

AP - Altura da Planta (cm); DDC - Diâmetro da Copa (cm); APB - Altura da Primeira Bifurcação (cm); DCL - Diâmetro do Caule (cm); CFL - Comprimento da Folha (cm); LDF - Largura da Folha (cm); CDC - Comprimento da Corola (cm); CANT - Comprimento da Antera $(\mathrm{cm})$; CES - Comprimento do Estilete (cm); LAP - Largura da Pétala $(\mathrm{cm})$. Médias seguidas pela mesma letra, em cada coluna, pertencem a uma mesma classe, de acordo com o critério de Scott-Knott $(\mathrm{p}<0,01)$. 
Tabela 3. Agrupamento de 54 plantas de Capsicum annuum conforme método de Tocher

\begin{tabular}{ll}
\hline GRUPO & PLANTA \\
\hline 1 & $11,12,10,15,13,25,7,9,6,14,2,3,42,34,31$, \\
& $28,46,52,50,24,32,45,29,4,23,53,1,41,44$, \\
& $51,26,54,39,8,40$ \\
2 & $17,19,21,36,37,33,43,48$ \\
3 & $47,49,18,20$ \\
4 & 5,22 \\
5 & 27,38 \\
6 & 35 \\
7 & 30 \\
8 & 16 \\
\hline
\end{tabular}

Tabela 4. Contribuição relativa dos caracteres para diversidade Singh (1981)

Distância Generalizada de Mahalanobis

\begin{tabular}{lc}
\hline Variáveis & Valor \% \\
\hline Altura da planta & 4,58 \\
Diâmetro da copa & 9,22 \\
Altura da primeira bifurcação & 6,76 \\
Diâmetro do caule & 68,97 \\
Comprimento da folha & 1,80 \\
Largura da folha & 1,03 \\
Comprimento da corola & 1,80 \\
Comprimento da antera & 4,47 \\
Comprimento do estilete & 0,85 \\
\hline
\end{tabular}

O diâmetro do caule apresentou a maior variabilidade entre plantas, resultando em 13 classes, seguido de diâmetro da copa com oito, e altura da primeira bifurcação, com seis classes. A altura da planta resultou em cinco classes, seguida do comprimento da corola, com quatro. $\mathrm{O}$ comprimento da folha e a largura da pétala resultaram em três classes. A largura da folha e o comprimento do estilete resultaram em duas classes (Tabela 2). Resultados semelhantes foram encontrados por Barroso et al. (2012) e Nascimento et al. (2012), também em estudo de caracterização fenotípica de geração segregante de $C$. аппиит. A detecção da variabilidade existente dentro da família, aliada à dos altos valores de herdabilidade encontrados, permitem a obtenção de ganhos ao se praticar a seleção em geração precoce. Nascimento et al. (2012) e Barroso et al. (2012), trabalhando com diferentes gerações segregantes de $C$. аnnиum, verificaram comportamento semelhante, nas $\mathrm{F}_{2}$ avaliadas.

As plantas com menor altura foram 4, 10, 12, 17, 19, 24, $33,3743,48$ e 53, podendo ser selecionadas para avanço de geração e seleção de linhagens de porte muito baixo, uma vez que suas alturas variaram de 14 a $17 \mathrm{~cm}$. A planta 48, além de porte mais baixo, também apresenta o menor diâmetro da copa. Sua copa, entretanto, apresentou valor de diâmetro bem superior $(20 \mathrm{~cm})$ aos encontrados por Nascimento et al. (2012) e Barroso et al. (2012), para diâmetro da copa, 4 e $7 \mathrm{~cm}$, respectivamente. Barroso et al. (2012) argumentam que a harmonia entre o arquitetura da planta e o tamanho do vaso é importante para determinação da qualidade de uma pimenteira ornamental, sendo que a altura da planta e o diâmetro da copa devem ser de 1,5 a 2 vezes o tamanho do vaso. Neste caso, o tamanho ideal seria entre 22,5 e 26,5, para a altura da planta, e de 24 a 27, para o diâmetro, uma vez que, os vasos utilizados neste estudo tinham $16 \mathrm{~cm}$ e $13 \mathrm{~cm}$, de diâmetro e altura, respectivamente. Levando-se em consideração esse critério, várias linhagens podem ser selecionadas a partir deste estudo.

A planta 30 apresentou o maior comprimento do caule $(0,89 \mathrm{~cm})$. Esta característica é de interesse no melhoramento, uma vez que, plantas com caule muito fino tendem a acamar e perdem seu valor comercial. Entretanto, a família estudada por Nascimento et al. (2012) apresentou valores bem superiores, $2,79 \mathrm{~cm}$, aos encontrados neste trabalho para esta característica, o que pode ser explicado pela presença de segregação transgressiva.

O maior valor encontrado para largura de corola foi de $1,81 \mathrm{~cm}$, na planta 5 , não diferindo, estatisticamente, dos valores obtidos para as plantas $3,7,11,13,14,15$ e 19 . Esses valores, entretanto, são menores do que aqueles encontrados por Nascimento et al. (2012) e Santos et al. (2013), de 2,29 e 2,18 cm, respectivamente. Estes últimos autores concluíram, em seu trabalho, que é possível criar novas variedades de pimenteiras com flores maiores, o que não se aplica para a família estudada neste trabalho.

Quando se utilizaram as técnicas multivariadas, foi possível agrupar as 54 plantas em oito grupos, sendo o grupo 1 o que agrupou o maior número de plantas (35), seguido dos grupos 2 e 3 , com oito e quatro plantas, respectivamente. Os grupos 4 e 5 foram compostos por duas plantas cada, e os grupos 6, 7 e 8 foram compostos por apenas uma planta (Tabela 3).

A característica que mais contribuiu para a divergência genética foi o diâmetro do caule $(68,97 \%)$, seguida pelo diâmetro da copa $(9,22 \%)$, altura da primeira bifurcação $(6,76 \%)$ e altura da planta $(4,58 \%)$. Somadas, essas características de planta contribuíram com $89,53 \%$ da variabilidade detectada. Já as características de flor contribuíram apenas com 10,47\%, sendo que só o comprimento da antera contribuiu com apenas $4,48 \%$, tendo sido a característica floral com maior peso (Tabela 4). Ao contrário do que se observou neste estudo, Rêgo et al. (2011c), trabalhando com a espécie $C$. baccatum, observaram que as características de fruto foram as que mais contribuíram para a divergência genética, enquanto as variáveis relacionadas com o porte, citadas acima, foram as que menos 
contribuíram para a diversidade da espécie em estudo. $\mathrm{O}$ fato de essas características terem contribuído mais para a variabilidade, neste estudo, evidencia que há grande variabilidade na população base, o que a torna útil para programas de seleção.

\section{CONCLUSÃO}

É possível praticar seleção dentro desta família, para dar continuidade ao Programa de Melhoramento de Pimenteiras Ornamentais, em vista da variabilidade encontrada.

As características que mais contribuíram para a divergência genética foi o diâmetro do caule, o diâmetro da copa, a altura da primeira bifurcação e altura da planta. Somadas, essas características de planta contribuíram com $89,53 \%$ da variabilidade detectada.

Apenas o comprimento da antera apresentou baixa herdabilidade $(22,25 \%)$. As demais variáveis analisadas apresentaram valores de herdabilidade acima de $73 \%$. Estas últimas características podem ser selecionadas já em gerações precoces devido aos altos valores de herdabilidade encontrados.

A seleção para o comprimento da antera não será eficiente, uma vez que esta característica não apresentou variabilidade entre as plantas.

\section{REFERÊNCIAS}

Barroso PA, Rêgo ER, Rêgo MM, Nascimento KS, Nascimento NFF, Nascimento MF, Soares WS, Ferreira KTC \& Otoni WC (2012) Analysis of segregating generation for components of seedling and plant height of pepper (Capsicum annuum L.) for medicinal and ornamental purposes. Acta Horticulturae, 953:269-276.

Cruz CD (2008) Programa Genes - Diversidade Genética. Viçosa, UFV. 278p.

IPGRI - International Plant Genetic Resource Institute (1995) Descritores para Capsicum spp. Roma, IPGRI. 51p.

Finger FL, Rêgo ER, Segatto FB, Nascimento NFF \& Rêgo MM (2012) Produção e potencial de mercado para pimenta ornamental. Informe Agropecuário, 33:14-20.

Marim BG, Silva DJH, Carneiro PCS, Miranda GV, Mattedi AP \& Caliman FRB (2009) Variabilidade Genética e Importância Relativa de caracteres em acessos de germoplasma de tomateiros. Pesquisa Agropecuária Brasileira, 44:1283-1290.

Nascimento NFF, Rêgo ER, Nascimento MF, Finger FL, Bruckner CH, Silva Neto JJ \& Rêgo MM (2012) Heritability and variability of morphological traits in a segregating generation of ornamental pepper. Acta Horticulturae, 953:299-304.

Ohara R \& Pinto CMF (2012) Mercado de Pimentas Processadas. Informe Agropecuário, 33:07-13.

Rêgo ER, Finger LF \& Rêgo MM (2011a) Produção, Genetica e Melhoramento de Pimentas (Capsicum spp.). Areia, Universidade Federal da Paraíba. 223p.

Rêgo ER, Finger FL \& Rêgo MM (2011b) Types, Uses and Fruit Quality of Brazilian Chili Peppers. In: Kralis JF (Ed) Spices: Types, Uses and Health Benefits. New York, Nova Science Publishers. p.01-70.
Rêgo ER, Rêgo MM, Cruz CD, Finger FL \& Casali VWD (2011c) Phenotypic diversity, correlation and importance of variables for fruit quality and yield traits in Brazilian peppers (Capsicum baccatum).Genetic Resources and Crop Evolution, 58:909-918.

Rêgo ER, Rêgo MM, Silva DF, Santos RMC, Sapucay MJLC, Silva DR \& Silva Júnior SJ (2009) Selection for leaf and plant size and longevity of ornamental peppers (Capsicum spp.) grown under greenhouse condition. Acta Horticulturae, 829:371-374.

Rêgo ER, Silva DF, Rêgo MM, Santos RMC, Sapucay MJLC \& Silva DR (2010) Diversidade entre linhagens e importância de caracteres relacionados à longevidade em vaso de linhagens de pimenteiras ornamentais. Revista Brasileira de Horticultura Ornamental, 16:165-168.

Rêgo ER, Finger FL \& Rêgo MM (2012a) Consumption of pepper in Brazil and its implicarions on nutrition and health of humans and animals. In: Peppers: Nutrition, Comsumpion and Health. New York, Nova Science Publishers. p.159-170.

Rêgo ER, Santos RMC, Rêgo MM, Nascimento MF, Nascimento NFF \& Silva AM (2012b) Produção de mudas e disponibilização de cultivares de pimenteiras: sustentabilidade, inclusão social e geração de trabalho e renda nas comunidades de Macacos e Furnas no brejo paraibano. In: Miranda VCM, Sobrinho RGS \& Rêgo ER (Eds.) Sustentabilidade, Inclusão Social e Geração de Trabalho e Renda - Perspectivas de Extensão Universitária. Areia, Universidade Federal da Paraíba. p.11-30.

Rêgo ER, Nascimento MF, Nascimento NFF, Santos RMC, Fortunato FLG \& Rêgo MM (2012c) Testing methods for producing self-pollinated fruits in ornamental peppers. Horticultura Brasileira 30:669-672.

Rêgo ER, Rêgo MM, Costa FR, Nascimento MF, Nascimento NFF, Barbosa LA, Fortunato FLG \& Santos RMC (2012d) Analysis of diallel cross for some vegetative traits in chili pepper. Acta Horticuture, 937:297-304.

Santos RMC, Rêgo ER, Nascimento MF, Nascimento NFF, Rêgo MM, Borém A, Finger FL \& Costa DS (2012) Ethylene resistance in a $\mathrm{f}_{2}$ population of ornamental chili pepper (Capsicum annиит) Acta Horticulturae, 1000:433-438.

Santos RMC, Rêgo ER, Borém A, Nascimento NFF, Nascimento MF, Finger FL, CarvalhoGC, Lemos RC \& Rêgo MM (2013) Ornamental Pepper Breeding: Could a Chili be a Flower Ornamental Plant. Acta Horticulturae, 1000: 451-456.

Singh D (1981) The relative importance of characters affecting genetic divergence. Indian Journal of Genetics \& Plant Breed, $1: 237-245$.

Sreelathakumary I \& Rajamony L (2004) Variability, heritability and genetic advance in chilli (Capsicum annuum). Journal of Tropical Agriculture, 42:35-37.

Stommel JR \& Bosland PW (2007) Ornamental pepper, Capsicum annuum. In: Flower AN (Ed.) Breeding and Genetics: Issues, Challenges and opportunities for the $21^{\text {st }}$ Century. Dordrecht, Springer. p.561-599. 